\title{
Influence of Design Features of Double- Circuit Steeply Inclined Conveyors on Their Operational Parameters and Performance
}

ISSN: 2578-0255

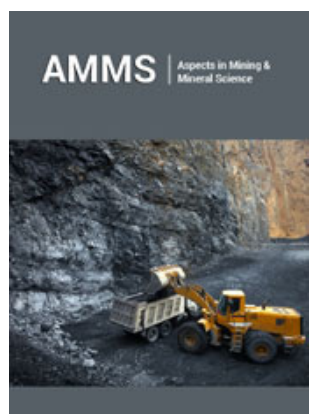

*Corresponding author: : Glebov Andrey Valerjevich, Candidate of engineering sciences, Deputy director on scientific questions, Federal state budget establishment the Institute of Mining, Ural branch Russian academy of sciences (IM UB RAS), Russia

Submission: 漹July 26, 2019

Published: : 1 ingust 12, 2019

Volume 3 - Issue 3

How to cite this article: Glebov AV, Vladimirovich SA, Anatoljevich BV, Saginovich KS, Influence of Design Features of Double-Circuit Steeply Inclined Conveyors on Their Operational Parameters and Performance.Aspects Min Miner Sci.3(2). AMMS.000562.2019.

DOI: 10.31031/AMMS.2019.03.000562

Copyright@Glebov AV, This article is distributed under the terms of the Creative Commons Attribution 4.0 International License, which permits unrestricted use and redistribution provided that the original author and source are credited.

\author{
Glebov AV ${ }^{1 *}$, Semenkin AV ${ }^{1}$, Bersenev $\mathrm{VA}^{1}$ and Kulniyaz $\mathrm{SS}^{2}$
}

${ }^{1}$ Federal state budget establishment the Institute of Mining, Ural branch Russian academy of sciences (IMUB RAS), Russia

${ }^{2}$ Aktobe regional state University. K. Zhubanova, Kazakhstan

\begin{abstract}
Some results of the comparison of the CLS with moving clamping elements and clamping elements fixed on the line of the conveyor are given. The assessment was carried out on the main limiting parameters for hourly cargo traffic in the range of 1000-6000t/h using rubber cable belts. The advantages of steeply inclined conveyors with moving clamping elements based on the specifics of the operation of conveyor belts in the transition section are shown. As a result of the study of possible types of wear of conveyor belts, it has been proposed to use the formula for estimating the duration of their service, which determines the duration of the belts operation on traditional belt conveyors transporting coarse rock mass. For conditional conveyor, considered structures calculated service life of conveyor belts. The cost comparison of conveyor belts showed that the ratio of initial costs and operating costs of conveyor belts of high-inclined conveyors with moving clamping elements to the corresponding costs and expenses of high-inclined conveyor belts with stationary clamping elements for a conditional conveyor is 0.87 and 0.65 respectively.
\end{abstract}

Keywords: Dual steeply inclined conveyor; Load carrying belt; Hoisting belt; Mechanical clamping device; Moving clamping elements with resilient-elastic properties; Corrugation; Inclination angle; Traditional belt conveyor; The cost of transportation

Analysis

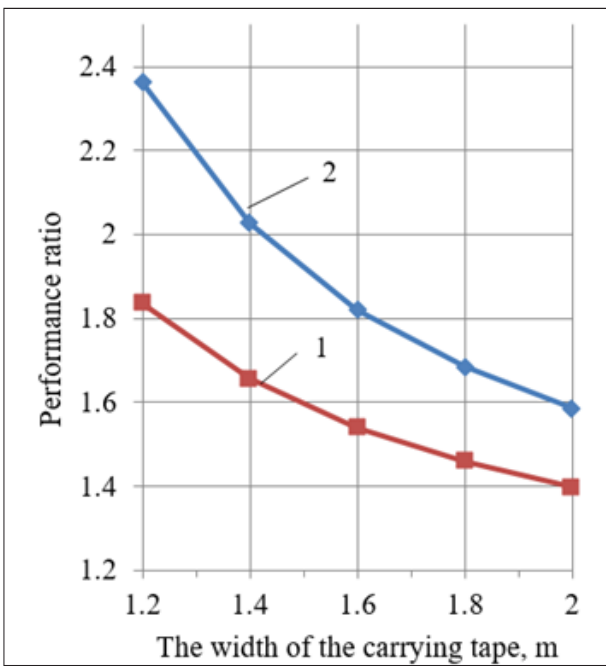

Figure 1: The ratio of the theoretical time performance of the CLS with moving clamping elements $\mathrm{Qдв,} \mathrm{depending} \mathrm{on} \mathrm{the} \mathrm{width} \mathrm{of} \mathrm{the} \mathrm{load-carrying} \mathrm{tape:} \mathrm{1-at} \mathrm{the}$ width of the clamping edge at the KNKst $b \sigma=150 \mathrm{~mm}, 2$-the same at $b \sigma=200 \mathrm{~mm}$

The increasing complexity of mining conditions in the development of deep-seated mineral deposits by the open method necessitates the use of steeply inclined lifts in quarries for the delivery of rock mass to the surface.In the structures of the mechanization of cyclic-flow technology (CLT), which is the most effective in the development of deep quarries, the use of steeply inclined belt conveyors (CLS) is preferable. By analyzing the design of steeply inclined 
conveyors, a large variety of their structures was established for various operating conditions [1-9]. In deep quarries, it is advisable to use double-circuit conveyor belts with pressure (load-holding) belts (inclination angle up to $25-30^{\circ}$ ) and additional load pressing using devices interacting with cargo-holding tape (lifting angle more than $30^{\circ}$ ) $[10,11]$. Clamping devices in the form of springloaded or sprung rollers mounted permanently on a linear conveyor stand were widely used abroad. They press the side strips of the load-bearing and load-holding tapes to each other and the last to the transported material, creating the necessary clamping force (Figure 1).

High-angle conveyors with the above-mentioned clamping devices have significant drawbacks [12]:

a) permanently installed clamping devices of the load carrying tape create prerequisites for the movement of the transported material between them in the direction of the tail part of the conveyor with uneven loading of the tape. This may occur due to additional rolling forces arising from the interaction of pieces of the transported material with the clamping elements. This phenomenon reduces the reliability and safety of the conveyor;

b) the resulting dynamic loads during the interaction of the clamping devices with pieces of the transported material significantly increase its movement, which significantly increases the wear of the working surfaces of conveyor belts. The wear of the belts is also increased due to additional bending deformations in the section of the transition curve of the tail part of the conveyor, arising in the belts due to a change in the shape of their cross section;

c) reduction of the receiving capacity of the load-carrying tape to $30-40 \%$ due to the use of its side strips with a width of about $200-250 \mathrm{~mm}$ to press the load-holding tape with side rollers.

There is a proposal for the implementation of clamping elements of the CLS, moving synchronously with the tape of the clamping circuit [13]. A feature of the design is the execution of clamping elements in the form of corrugations, mounted on the outer (working) surface of the load-carrying circuit tape [14]. The corrugations are made of an elastic material, have a good damping ability and restore their shape after the disappearance of contact with the transported material. The maximum height of the corrugation corresponds to the depth of the load-carrying tape chute, and in the absence of rock mass on it, the corrugation enters its chute and performs the function of a partition. Such a design of the conveyor allows: to fully utilize the width of the load-carrying belt, as in traditional belt conveyors; to eliminate additional bending deformations of the load carrying and pressure tapes, as well as reduce the stresses in them at the section of the transition curve; reduce the movement of the transported material on the line of the conveyor. These factors significantly improve the operational performance of a steeply inclined lift of the rock mass in comparison with the CLS with stationary clamping elements. The following are some of the results of the comparison of the CLS with moving clamping elements and clamping elements that are permanently mounted on the line of the conveyor. The assessment was carried out on the basic limit parameters of the CLS for hourly cargo traffic in the range of 1000-6000t/h using rubber cable belts with a speed of $3.15 \mathrm{~m} / \mathrm{s}$ and lifting the rock mass with a bulk density of $2 \mathrm{t} / \mathrm{m} 3$ at an angle of $45^{\circ}$. Comparison of performance was carried out on the hourly traffic, determined when using load-carrying tapes of the same width $(1200-2000 \mathrm{~mm})$ for the CLS of the considered designs. For other important factors (operation of conveyor belts on sections of transition curves, their deformation when cargo is pressed, relative slippage of load carrying and cargo holding bands due to possible desynchronization of their movement, etc.) that have a significant impact on wear and durability of conveyor belts due to lack theoretical studies and information on the practical operation of the CPC carried out only a qualitative assessment.

\section{Research}

For KNK with moving clamping elements, the authors have developed a schematic diagram and a method for determining the main parameters and performance indicators [12], which allows it to be enlarged to evaluate its advantages and disadvantages relative to CLS of other designs. The calculated basic parameters of the CLS with stationary and moving clamping elements are presented below (Table 1). $\mathrm{m}$ of the lifting height of the transported material $(\mathrm{kW} / \mathrm{m})$ differ slightly from the considered CLS. From the above information, it follows that for a CLS with stationary clamping elements, the total width of conveyor belts is 1.3-1.4 times greater than that of a CLS of a different design. In this case, the calculated strengths of the ribbons in both cases are equal. As a result, the maximum length of the conveyor and the height of the rock mass rise are 1.15-1.25 times. This indicator, in some quarrying conditions, when a conveyor elevator needs to be laid in one stage, can be crucial in choosing the design of the CLS. With regard to the power of the drive stations, with its larger total value for the CLS with stationary clamping elements, for the reason mentioned above, the specific values per 1 Comparison of a very important indicator, the performance of conveyor installations, is presented as the ratio of the estimated time performance of the CLS with moving clamping elements (Qдв) to the same value of the KNK with stationary pressure elements (QCT) with equal widths of loadcarrying tapes (Figure 1). When calculating the hourly productivity for a CLS with stationary clamping elements, the working width of the load-carrying tape was taken to be equal, taking into account the unloaded side lanes (bб ) with a width of 150 and $200 \mathrm{~mm}$. Given the ratio of productivity, decreasing with decreasing value of bб and increasing with its increase, largely characterizes the use of the technical capabilities of conveyor equipment. The theoretical coefficient of using technical capabilities, determined by the product of the coefficients of using equipment in time and in productivity, should be expected to be 1.6-2.35 times higher in a CLS with moving clamping elements than in a CLS of a different design with bб=200 $\mathrm{mm}$ and 14-1.8 times with bб $=150 \mathrm{~mm}$. This factor has a very significant effect on the cost of a conveyor lifting $1 \mathrm{t}$ of rock mass from the quarry and labor productivity. 
Table 1: Main parameters of double-circuit steeply inclined conveyors.

\begin{tabular}{|c|c|c|c|c|c|c|c|}
\hline \multirow{2}{*}{ Parameter Name } & \multicolumn{7}{|c|}{ Productivity, $\mathrm{t} / \mathrm{h}$} \\
\hline & 1000 & 2000 & 3000 & 4000 & 5000 & 6000 & \\
\hline \multirow{4}{*}{ Tape width, mm } & \multirow{2}{*}{ 1) } & $1200 * *)$ & 1600 & 1800 & 2000 & 2000 & 2200 \\
\hline & & 1200 & 1600 & 1800 & 2000 & 2000 & 2200 \\
\hline & \multirow{2}{*}{ 2) } & $1200 * *)$ & 1200 & 1400 & 1600 & 1600 & 1800 \\
\hline & & 1000 & 1000 & 1200 & 1400 & 1400 & 1600 \\
\hline \multirow{4}{*}{$\begin{array}{c}\text { Tape strength, } \mathrm{n} / \mathrm{mm} \\
\text { widths }\end{array}$} & \multirow{2}{*}{ 1) } & 4000 & 5000 & 5000 & 4000 & 4000 & 4000 \\
\hline & & 2500 & 2500 & 2500 & 2500 & 2500 & 2500 \\
\hline & \multirow{2}{*}{ 2) } & 4000 & 5000 & 4000 & 4000 & 4000 & 4000 \\
\hline & & 2500 & 2500 & 2500 & 2500 & 2500 & 2500 \\
\hline \multirow{2}{*}{ Maximum length of the conveyor, $\mathrm{m}$} & 1) & 714 & 610 & 421 & 355 & 284 & 260 \\
\hline & 2) & 725 & 429 & 333 & 287 & 231 & 217 \\
\hline \multirow{2}{*}{ Material lifting height, $\mathrm{m}$} & 1) & 510 & 430 & 298 & 250 & 200 & 183 \\
\hline & 2) & 516 & 303 & 236 & 203 & 163 & 153 \\
\hline \multirow{4}{*}{ Installed drive power, $\mathrm{kW}$} & \multirow{2}{*}{ 1) } & 1890 & 3000 & 3000 & 3750 & 3000 & 3750 \\
\hline & & 1000 & 1500 & 1500 & 1600 & 2000 & 2000 \\
\hline & \multirow{2}{*}{ 2) } & 1890 & 1890 & 2400 & 2600 & 2600 & 3000 \\
\hline & & 1000 & 1000 & 1250 & 1250 & 1250 & 1500 \\
\hline \multirow{2}{*}{ Power per $1 \mathrm{~m}$ lifting height, $\mathrm{kW} / \mathrm{m}$} & 1) & 5,7 & 10,46 & 15,1 & 21,4 & 25,0 & 31,42 \\
\hline & 2) & 5,67 & 9,54 & 15,46 & 18,96 & 23,6 & 29,4 \\
\hline
\end{tabular}

*) In item rock 1 shows figures for CLS with stationary clamping elements, in item rock 2-CLS for the moving clamping elements.

**) above the line there are indicators for the load-carrying contour, below the line for the load-carrying contour CLS.

In addition to the basic parameters of the CLS, the indicators characterizing the influence on the safety of operation of conveyor installations and the durability of their structural elements are important in their operation. One of them is the size of the concave transition section from the horizontal to the inclined part of the conveyor. The correct choice of parameters allows you to improve the conditions of retention of the transported material from slipping into the tail section of the CLS, reduce its possible spilling between the belts, reduce deformations and stresses in the conveyor belts, to some extent determine the length of the tail section of the conveyor, etc. When analyzing these indicators, you must first pay attention to the following. At the CLS, the lower branch of the load-carrying tape, after the passage of the last driving drum as it moves away from the drive station, may have a negative tension value, which increases towards the line of incident on the tail drum. This indicates an unacceptable sagging of the tape between the roller and the violation of its contact with the tail drum. To eliminate the slippage of the tape on the tail drum, it must have an appropriate tension. The required tension of the belt, depending on the length of the conveyor, the height of the load lifting and other conditions can reach $(25-30) \times 103 \mathrm{H}$. If the tensioning device is attached to the tail drum, large belt tensions are inevitable at the beginning and end of the transition curve of the conveyor, which is natural will increase its radius. In addition, the conveyor belt will not be fully utilized. These circumstances indicate that the main tensioning device of the conveyor, providing the necessary slack of the lower branch of the belt, is located in front of the tail drum, leaving its tension on it to the minimum possible. In this case, the tension and deformation of the tape on the transition curve, even at relatively small radii, will be minimally possible, which undoubtedly will have a positive effect on its wear and durability. When determining the radius of the concave transient curve, the CLS with stationary clamping elements EE. Sheshko draws attention to the stress state of conveyor belts moving along the transition section.

Studies have shown that the load-carrying tape when moving with a load under the action of a bending moment is subjected to tensile forces; clamping tape - as compressing (in the middle part), and stretching (on the edges). Since the tension of the belts on the load sections is significantly less (maximum) workers, there is no danger of overloading the load carrying tape in this mode. The limiting condition for justifying the radius of the transition curve can be only the compressive stresses in the pressure belt. In the modes of starting and stopping the conveyor, as well as when the conveyor is moving without a load, the load-carrying tape in the curvilinear section is pressed against the pressure belt under the action of tension and is also subjected to compressive and tensile stresses. The minimum radius of the transition curve is taken such that the tape will not experience any compressive or maximum tensile forces [15]. Analysis of the KNK scheme and the crosssection of its linear stalemate shows that at the beginning and end of the transition section the load-carrying and pressure tapes change the shape of the cross-section. At the beginning of the curve, the pressure belt has the shape of an inverted groove, created by three-roller rollers, and the load carrier takes the form of pressure tape, which it receives on a straight conveyor belt after the end of the transition section. On a straight inclined section after the 
transition, the load-carrying tape returns to its original position with groovedness as in the load area, and the pressure tape, taking the shape of the surface of the transported material, presses against it and to the side bands of the load-carrying tape with pressure rollers. As a result, the tapes experience additional alternating loads, which, to some extent, will increase wear and reduce their durability. In addition, it should be noted that the load-carrying tape on a curved section is not equipped with any devices that press it together with the transported material to the clamping tape. The rock mass in the grooved space between the belts is held only by the tension forces of the load-carrying belt. In this case, the resultant tension forces of the load-carrying tape at the beginning and end of the transition section should provide the lifting force of the tape with a slightly larger amount of resultant load created by the material being transported and the tape itself. Achievement of such tension of the load-carrying tape at the lowest possible tension on the tail drum of the conveyor is unlikely. To meet this condition, it is necessary to increase the preload of the load carrying belt, which to some extent will reduce the use of its traction capabilities and will affect the parameters of the conveyor installation. When using the above mentioned method of holding the transported material in the space between the tapes in the transition section, the course of the tapes on the linear belt of the conveyor should be almost perfect without deviations in any direction, which is further complicated by the change in their shape of channeling. In such conditions of operation of conveyor belts the spillage of the transported material is not excluded from the space between them.

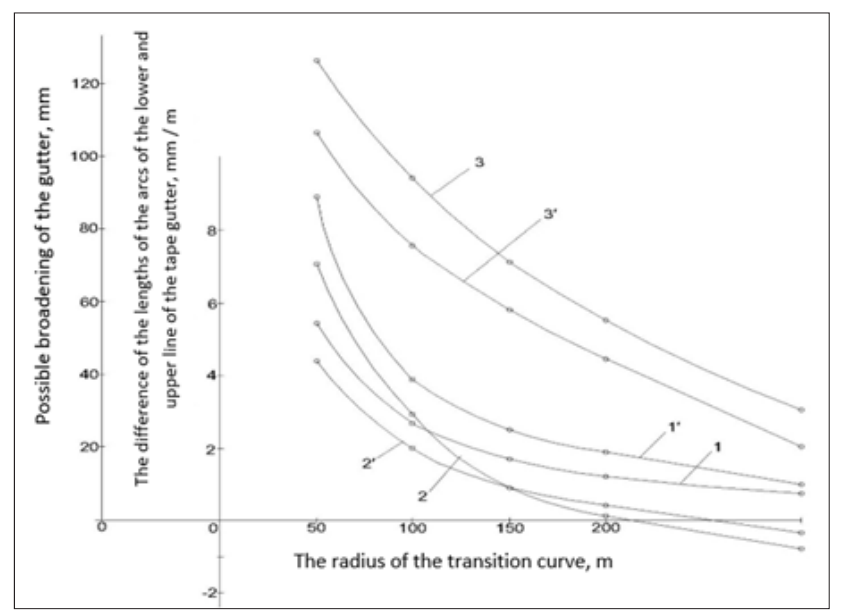

Figure 2: Indicators of the work of the CLS carrying tape on the transition section: 1-in idle mode with a tape width of $\mathrm{BT}=1200 \mathrm{~mm}, 1^{\prime}$-the same at $\mathrm{BT}_{\mathrm{T}}=1800 \mathrm{~mm}$; 2-in the working stroke mode with the productivity of the conveyor $\mathrm{Q}=1000 \mathrm{t} / \mathrm{h}, 2$ 'the same at $\mathrm{Q}=6000 \mathrm{t} / \mathrm{h}$; 3-broadening of the tape chute in idling mode at $\mathrm{BT}=1800 \mathrm{~mm}$, 3'-the same at $\mathrm{BT}_{\mathrm{T}}=1200 \mathrm{~mm}$.

Load-carrying conveyor belt KNK with moving clamping elements along the entire length of the conveyor moves with traditional groove. At the same time, the formation of cargo traffic is carried out at the loading point, equipped with roller rollers with the angle of inclination of the side rollers $30^{\circ}$, and after that, to improve the conditions for holding the load in the tape duct, the angle of inclination of the side rollers gradually rises to 45 $50^{\circ}$. A cargo holding belt consisting of a flat, performing the role of a traction, and a corrugated one fastened to it, to eliminate load spills on the line of the transitional and steeply inclined sections of the conveyor, is pressed against the sides of the load-carrying tape with the help of roller support. During the operation of the conveyor, the load-carrying belt under the action of the bending moment and the traction force at the transition section is subjected to compression and tension deformations due to the difference in the radii of the middle part and the lateral edges of the belt. To a greater extent, compressive strains experience the upper bands of the belt, forming its groove, and especially its beads. The difference in the lengths of the arcs of the middle part of the tape and its sides with a groove formed by roller supports with an angle of $45^{\circ}$ tilt of the side rollers reaches $5-8 \mathrm{~mm}$ per 1 meter of the transition section with a radius of $50 \mathrm{~m}$. roller guides will lose the shape of grooves with some variation in the width of the groove of the sides of the tape. This will entail additional flexural deformations and will somehow affect the belt durability. With an increase in the radius of the transition curve, the difference between the mentioned lengths of the arcs is significantly reduced and practically at the transition area with the radius of the curve $200-300 \mathrm{~m}$, the deformation of the compression of the ribbons becomes imperceptible. By reducing the grooving of the load-carrying tape in the roller-supports with the angle of inclination of the side roller $30^{\circ}$, it is not possible to avoid deformations of compression of the side edges of the tape with minimal tension on the tail drum. The difference in the lengths of the arcs of the middle part and the sides of the tape is $3.5-6.5 \mathrm{~mm}$ per 1 meter of the transition section with a radius of $50 \mathrm{~m}$.The extension of the load-carrying belt under operating loads with a conveyor capacity of $1000-6000 t / h$ partially reduces the compression deformations of its sides in the transition section with a radius of curves of $50-150 \mathrm{~m}$. Only when the radius of the transition curve is $250-300 \mathrm{~m}$ does the tape begin to experience tensile deformations. Increasing the preload of the load carrying belt will have little positive effect on the deformation of its sides but can significantly reduce the use of its traction capabilities. This is mainly due to the elastic properties of rubber cable conveyor belts, 
the relative elongation of which at full working load is $0.25 \%$, which corresponds to $2.5 \mathrm{~mm}$ per 1 meter of length. This problem can be solved by using high-strength conveyor belts with an elongation of $0.5-1 \%$. In this case, relatively small radii of transition curves are provided $(50-100 \mathrm{~m})$. For KNK with a small length and height of the transported material in one way, rubber-fabric tapes can be used, the relative elongation of which reaches $2-4 \%$. However, the limiting parameters of the CLS with such ribbons practically do not satisfy the mining conditions for the development of deep quarries in which the most appropriate use of a steeply inclined rise in the rock mass. Some indicators characterizing the operation of rubber cable conveyor belts in transition areas with different radii of curves are presented below (Figure 2). Due to the absence of grooving, the load-carrying conveyor belt of the KNK with moving clamping elements does not experience deformations of compression in almost all modes of operation of the conveyor belt. Therefore, it will not be a limiting condition in determining the radius of the transition curve.

Corrugated tape, bonded with traction, slightly increases the need for conveyor belts, but significantly affects the wear of the latter, perceiving all abrasive wear when in contact with the transported material on itself. In this regard, the durability of the traction belt is determined by its wear from bending deformations on the line and the drums, and corrugated wear from bending deformations and abrasive wear of the working lining of the tape. For corrugated ribbons, abrasive wear may increase due to some slippage between the load carrying and cargo holding ribbons, with possible desynchronization of their movement. In addition, a corrugated ribbon over time, there is a danger of stratification of the core of the corrugation. At the same time, the moving clamping element in the form of corrugations does not contribute to additional movement on the line and to the rolling back of the transported material, as is possible with a KNC with stationary clamping elements. Noted may reduce the abrasive wear of the load-carrying tape due to the greater stability of the traffic flow in its chute, including at desynchronization of the movement of tapes. As a disadvantage of using a cargo-holding tape with moving clamping elements in the CLS, in addition to the somewhat increased need for conveyor belts, we can note a more complicated initial installation due to the need to mount the corrugations along the entire length of the traction belt. But during operation, it is not possible to completely replace the corrugated tape, but only the most worn sections of it, which affects the maintainability of the equipment. To estimate the service life of conveyor belts of KNK with moving clamping elements, you can use the formula obtained by V.S. Volotkovsky as a result of the analysis of statistical data on the types of wear of conveyor belts and their duration of work at mining enterprises during the transportation of coarse rock mass [16]. The formula takes into account the wear of the belts at the loading point of the material, abrasive wear on the line and fatigue wear from bending stresses on the line and the drums of the conveyor. Additional wear of the ribbons during their relative slippage during the movement is proposed to take into account the coefficient introduced into the term, taking into account the abrasive wear of the ribbons on the line. This coefficient can be calculated by the expression:

$$
K_{c}=1+\frac{l_{c k}}{2 L_{\Gamma}}
$$

where Іск is the length of slippage per one revolution of the tape, $\mathrm{m}$;

$\mathrm{L} \Gamma$-the length of the carrying circuit of the conveyor, $\mathrm{m}$.

According to the developed method, the life expectancy of the CLS belts for the conveyor with a capacity of 3000t/h, the height of the rock mass with a bulk density of $2.0 \mathrm{t} / \mathrm{m} 3$ is $250 \mathrm{~m}$, with a radius of the transition curve of $100 \mathrm{~m}$. almost the same (about 15 months). Also, the same service life of the clamping belt of the CLS with stationary clamping elements and the corrugated strip of the cargo holding circuit of the CLA with moving clamping elements. The duration of their work is 1.3 times higher than that of the carrying tapes. The trailer belt of the cargo holding circuit of the CLS with moving clamping elements has a very long service life (more than 10 years). The obtained data on the service life allowed us to compare the costs of the CLS conveyor belts. For a conveyor with the parameters mentioned above, the ratio of the initial cost of conveyor belts of the CLS with moving clamping elements to the same value of the bands of the CLS with stationary clamping elements is 0.87 . This ratio is due to the need to use wider tapes for CLS with stationary clamping elements. A similar ratio of operating costs of the conveyor belt is 0.65 , which is largely due to the high service life of the traction belt of the load-carrying circuit. An important point in determining the radius of the transition section in the tail part of the conveyor is to ensure the movement of the loadcarrying tape along the transition roller rollers, while maintaining the grooviness, in any modes of the conveyor. Such modes include start, movement and braking without load (idle), start, movement and braking of the loaded conveyor (working stroke), as well as movement with uneven traffic in the groove of the load-carrying belt. The latter is intermediate between idle and working stroke, when certain sections of the load-carrying tape may be unloaded with a rock mass. This is ensured when the total tearing-off force of the tape from the rollers, which is directed along the radius of the transition curve and determined by the tension-carrying forces of the load-carrying tape at the ends of the transition section, will be balanced by the forces that press the tape against the roller supports. During a working stroke, these forces are determined by the mass of the load carrying and clamping belts, the mass of the transported material along the arc of the transition curve and the clamping force generated by the clamping devices. To clarify this point, traction calculations were carried out, which determine the tension of the load-carrying tape at the beginning and end of the transition section of the CLS with moving clamping elements at hourly productivity of 1000 and 6000 tons and change of the radii of the transition curves in the range of $50-300 \mathrm{~m}$. the tail drum was assumed to be $1000 \mathrm{daN}$. As a result of the calculations, the power characteristics of the load-carrying KNK tape were obtained: tension of the tape at the beginning and end of the transition section; the resultant of these tensions; force tending to lift the tape over the roller carriages of the linear rod in the transition section 
of the conveyor (lifting force); the resultant loads on the roller supports from the load-carrying contour and the linear load on the load-holding contour (Table 2). The obtained calculated values of the indicators indicate that in the working stroke mode, a stable movement of the load-carrying loop of the belt along the roller supports of the linear section of the transition section is ensured. The load only from the working branch of the tape quite significantly exceeds the force of lifting the tape from the roller-support. The ratio of these indicators increases with increasing radius of the transition curve. The graphical interpretation of these quantities in the form of a linear dependence clearly illustrates this position (Figure 3). In idle mode, the tension of the load carrying tape at the extreme points of the transition curve is significantly reduced (4-7 times with a conveyor capacity of $6000 \mathrm{t} / \mathrm{h}$ ). In proportion to the decrease in the tension of the tape, the forces of lifting it above the roller supports decrease, which ensures the normal movement of the load-carrying tape in the transition curve.

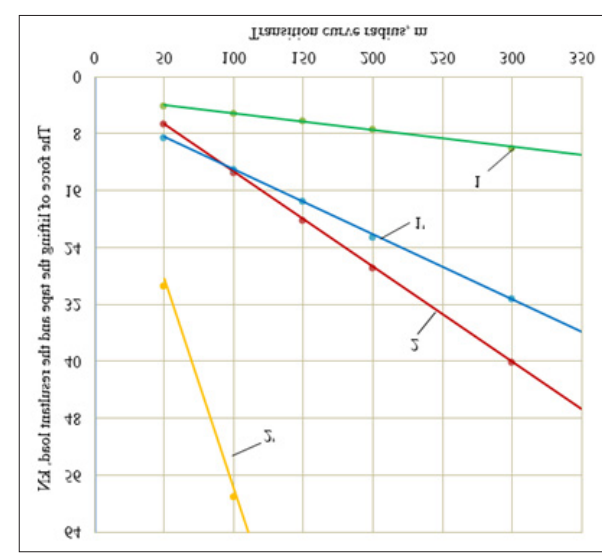

Figure 3: Change of the lifting force of the belt from the rollers (line 1 -at $Q=1000 t / h, 1$ '-at $Q=6000 t / h$ ) and the resultant load of the load-carrying circuit (line 2-at $\mathrm{Q}=1000 \mathrm{t} / \mathrm{h}, 2$ '-at $\mathrm{Q}=6000 \mathrm{t} / \mathrm{h}$ ) depending on the radius of the transition curve.

Table 2: The power characteristics of the load carrying tape on the transition section of the KNK.

\begin{tabular}{|c|c|c|c|c|c|c|c|c|c|}
\hline \multicolumn{10}{|c|}{ Parameters and Indicators } \\
\hline \multirow{2}{*}{$\begin{array}{c}\text { Productivity, } \\
\text { t/h }\end{array}$} & \multirow{2}{*}{$\begin{array}{c}\text { Transition } \\
\text { curve radius, } \\
\mathrm{m}\end{array}$} & \multirow{2}{*}{$\begin{array}{c}\text { Transition } \\
\text { curve length, } \\
\mathrm{m}\end{array}$} & \multicolumn{2}{|c|}{ Long load, daN / m } & \multirow{2}{*}{$\begin{array}{c}\begin{array}{c}\text { Equivalent } \\
\text { load }\end{array} \\
\begin{array}{c}\text { load- } \\
\text { carrying } \\
\text { circuit daN }\end{array}\end{array}$} & \multicolumn{2}{|c|}{ Stretching the tape, daN } & \multirow{2}{*}{$\begin{array}{c}\text { Equivalent } \\
\text { belt tension, } \\
\text { daN }\end{array}$} & \multirow{2}{*}{$\begin{array}{l}\text { Belt lifting } \\
\text { force, daN }\end{array}$} \\
\hline & & & $\begin{array}{l}\text { load } \\
\text { carrying } \\
\text { circuit }\end{array}$ & load loop & & $\begin{array}{l}\text { at the } \\
\text { beginning of } \\
\text { the curve }\end{array}$ & $\begin{array}{l}\text { at the end of } \\
\text { the curve }\end{array}$ & & \\
\hline \multirow{5}{*}{1000} & 50 & 39,2 & & & 6672 & 4009 & 6727 & 4815 & 4105 \\
\hline & 100 & 78,5 & $170,2 x)$ & $78 x)$ & 13361 & 4009 & 9446 & 7193 & 5149 \\
\hline & 150 & 118 & 60 & 58 & 20084 & 4009 & 12163 & 9749 & 6198 \\
\hline & 200 & 157 & & & 26781 & 4009 & 14881 & 12375 & 7377 \\
\hline & 300 & 235,5 & & & 40082 & 4009 & 22018 & 19392 & 9987 \\
\hline \multirow{5}{*}{6000} & 50 & 39,2 & & & 29451 & 5173 & 17016 & 13851 & 8490 \\
\hline & 100 & 78,5 & 751,3 & 255 & 58977 & 5173 & 28859 & 25466 & 13040 \\
\hline & 150 & 118 & 89 & 93 & 84145 & 5173 & 40700 & 37223 & 17533 \\
\hline & 200 & 157 & & & 117954 & 5173 & 52544 & 50158 & 22537 \\
\hline & 300 & 235,5 & & & 176931 & 5173 & 76236 & 72665 & 31169 \\
\hline
\end{tabular}

$\mathrm{x})$ is the load: above the line-for the working stroke, below the line-for idling.

\section{Research Results}

\section{The study found}

a) With the same hourly cargo traffic, the limiting parameters of the CLS with stationary clamping elements (the maximum length of the conveyor and the lifting height) are 1.15-1.25 times the value of these parameters for the CLS with moving clamping elements. This is connected with the use of wider ribbons due to the necessity of pressing the side bands $150-250 \mathrm{~mm}$ wide for the load carrying and clamping bands of the space station with stationary pressure elements. The total width of the strips of these CLS s is 1.3-1.4 times larger than that of a CLS with moving clamping elements. At the same time, the specific power required for lifting 1 ton of rock mass per $1 \mathrm{~m}$ of lifting height for CLS of both designs is almost the same and varies in the range of 5.7-31.5 $\mathrm{kW} / \mathrm{m}$ depending on the hourly productivity of the conveyor.

b) With an equal width of the load-carrying tapes of the compared CLS, a conveyor with moving clamping elements has a significant advantage in clock performance. Its calculated value is 1.4-1.8 times higher than that of a CLS of a different design (with a width of an unloaded side band of load-carrying tape $b b=150 \mathrm{~mm}$ ) 
and 1.6-2.35 times (with $b b=200 \mathrm{~mm}$ ). As a consequence, the utilization rate of the technical capabilities of the equipment with a CLS with moving clamping elements should be expected 1.4-1.8 times higher than that of a CLS with stationary clamping elements with $\mathrm{bb}=150 \mathrm{~mm}$ and $1.6-2.35$ times with $\mathrm{bb}=200 \mathrm{~mm}$.

c) To make full use of the possibility of the conveyor belt when choosing the radius of the transition curve, it should be borne in mind that the movement of the load-carrying belt on the tail drum was without slipping with the minimum allowable tension. This is ensured by the location of the tensioner, which eliminates sag of the lower branch of the load-carrying tape on the roller supports, in front of the tail drum.

d) Based on the specifics of the work of conveyor belts in the transition section, the prefabricated complex with moving clamping elements has preference. Analysis has shown that, at this conveyor, the load-carrying tape does not experience alternating deformations in the transition section due to the constancy of the cross-sectional shape over the entire length. This ensures the absence of spillage of the transported material and additional tape wear. In addition, the conditions for retaining the cargo in the tape chute are improved due to its stable movement. Undesirable is the possible broadening of the gutter of the load-carrying tape between the roller carriers of the transition section. Part of this issue can be solved by using highstrength conveyor belts with relative elongation at an operating load of $0.5-1 \%$. Due to the lack of grooving in all modes of operation of the conveyor, the load holding belt does not experience any compression deformations on the line. Therefore, it will not be a limiting condition in determining the radius of the transition curve. Checking the stability of the movement of the load-carrying tape along the roller-supports of the transition section showed that the loads pressing the load-carrying tape as it moves with and without the load exceed significantly the resultant forces seeking to lift the tape over the roller-supports, with all the transition radii and capacities considered. Consequently, this factor is unlikely to affect the choice of the radius of the transition curve.

As a result of the analysis of possible types of wear of conveyor belts, CLS proposed to use the formula for estimating their service life, which determines the duration of belts operation on traditional belt conveyors transporting coarse rock mass. At the same time, correction factors are introduced into the formula, taking into account the wear due to slippage of the ribbons relative to each other and additional bending deformations that occur when the tape hollowness changes in the transition section. For conditional conveyor, considered structures calculated service life of conveyor belts. The calculations found that the service life of the load carrying tapes of both CLS is almost the same. Also, the service life of a clamping belt with a stationary clamping element and a corrugated strip of a cargo-holding circuit with moving clamping elements is almost equal. The duration of their work is 1.3 times higher than that of the carrying tapes, which is associated with the absence of wear at the loading point of the rock mass. The traction belt of the cargo holding circuit of the CLS with moving clamping elements has a rather high service life (more than 10 years), since it undergoes mainly bending deformations. The cost comparison of conveyor belts showed that the ratio of initial costs and operating costs of conveyor belts of the CLS with moving clamping elements to the corresponding costs and expenses of the CLS belts with stationary clamping elements for the conditional conveyor is 0.87 and 0.65 , respectively.

\section{References}

1. Nikolaev ED (1998) Analysis of designs of steeply inclined conveyors for quarries. Mining Journal 11(12): 62-25.

2. Grebeneshnikov AL The development of technology steeply inclined conveyors. C FLEXOWELL $\AA$ and KNK POCKETLIFT $®$

3. Burr AN (2006) High-angle conveyor belts for the mining industry. Mining Equipment and Electrical Engineering 10: 22-26.

4. Burr AN (2007) Problems of application of various types of steeply inclined belt conveyors. Heavy Engineering 3: 31-34.

5. Sheshko EE (2008) Problems of steeply inclined belt conveyors with a clamping belt. Mining Machines: Sat scientific papers 8: p. 384.

6. Burr AN (2003) Prospects for the use of steeply inclined conveyors with a clamping belt at the center. Mining Journal 6: 52-56.

7. Nikolaev ED, Nazarov AV (2010) New technology for the development of deep quarries with the use of upgraded steeply inclined conveyors. Subsoil Use XXI Century 2: 62-64.

8. Perten Yu A (1977) Steeply inclined conveyors. Mechanical Engineering.

9. Nikolaev ED (1988) Analysis of steeply inclined conveyor designs for deep quarries. Mountain log 11-12: 62-65.

10. Kotyashev AA, Kaledin AV (1990) The use of belt steeply inclined conveyors for the transportation of rock mass. Mining Journal 5: 61-63.

11. Sheshko EE, Morozov VI, Burr NG (1996) Prospects for steeply inclined conveyor lifting at mining enterprises. Mining Journal 6: 56-59.

12. Karmaev GD, Glebov AV (2012) The choice of mining equipment cyclical flow technology of quarries. Ekaterinburg: IGD UB RAS, Russia. p. 296.

13. A patent for a steeply inclined conveyor with moving clamping elements was obtained instead of a.s. 1759754 Belt conveyor Bull. No. 33 07.09.92.

14. Glebov AV, Karmaev GD, Bersenev VA (2018) Innovative engineering design of deep-seated mineral deposits. Aspects in Mining and Mineral Science 2(2): 535

15. Sheshko EE (2008) Problems of steeply inclined belt conveyors with pressure belt in open pits.

16. Volotkovsky VS, Nokhrin EG, Gerasimova MF (1976) Wear and durability of conveyor belts. 